\title{
Correlation Between GAP43 and Nerve Growth Factor Receptors in Rat Sensory Neurons
}

\author{
V. M. K. Verge, ${ }^{1}$ W. Tetzlaff, ${ }^{2}$ P. M. Richardson, ${ }^{1}$ and M. A. Bisby ${ }^{3}$ \\ 'Division of Neurosurgery, Montreal General Hospital and McGill University, Montreal, Canada H3G 1A4, ${ }^{2}$ Department of \\ Medical Physiology, University of Calgary, Alberta, Canada T2N 4N1, and 'Department of Physiology, Queen's University, \\ Kingston, Ontario, Canada K7L 3N6
}

In mature rat sensory neurons, expression of the gene for the growth-associated protein, GAP43, was studied by in situ hybridization with a cDNA probe. Among neurons in normal lumbar dorsal root ganglia, labeling for GAP43 mRNA was heterogeneous, approximately one-half of the neurons being densely labeled. To characterize the latter population, individual neurons were examined in adjacent sections processed either for GAP43 hybridization or NGF-receptor radioautography. Virtually all neurons with high-affinity NGF binding sites had high basal levels of GAP43 mRNA and most GAP43-positive neurons bore NGF receptors. Another NGFresponsive population, sympathetic neurons in the superior cervical ganglion, also had high basal concentrations of GAP43 mRNA. Further co-localization studies in dorsal root ganglia were performed with immunohistochemistry for somatostatin and enzyme histochemistry for acid phosphatase. The latter 2 groups of sensory neurons have been previously shown to lack high-affinity receptors and were here shown to have low basal concentrations of GAP43 mRNA. From this and earlier studies, it can be assumed that substance P-immunoreactive neurons and strongly positive CGRP neurons synthesize GAP43 at high basal rate. One week following peripheral nerve transection, almost all neurons had high concentrations of GAP43 mRNA without correlation with NGF binding. Intrathecal infusion of NGF after the sciatic nerve was cut did not strongly influence this posttraumatic elevation in GAP mRNA.

In normal dorsal root ganglia, neurons that have high-affinity NGF binding sites and are therefore potentially responsive to NGF also have high basal rates of synthesis of GAP43. As an early response to nerve injury, synthesis of GAP43 is increased in sensory neurons irrespective of whether they are NGF-responsive. The synthesis of GAP43 in sensory neurons appears to be regulated by NGF under normal conditions but by some alternative signal soon after axonal injury.

\footnotetext{
Received July 26, 1989; revised Sept. 25, 1989; accepted Sept. 27, 1989.

Supported by grants from M.R.C. (Canada), N.I.H., and the Rick Hansen Man in Motion Legacy Foundation (Canada) to P.M.R. and M.A.B. W.T. is a fellow of the Alberta Heritage Foundation for Medical Research and V.V. receives a stipend from the Canadian Paraplegic Association. We thank Dr. Pate Skene for generously providing us with his GAP43 cDNA clone and Dr. W. Tatton for the image-analysis software

Correspondence should be addressed to M. A. Bisby at the above address.

Copyright (C) 1990 Society for Neuroscience $0270-6474 / 90 / 030926-09 \$ 02.00 / 0$
}

Growth-associated protein GAP43 (also known as B50, F1, pp 46 and pp 57 , and neuromodulin) is widely considered to be involved in neuronal mechanisms underlying axonal growth, axonal regeneration, and synaptic plasticity (Benowitz and Routtenberg, 1987). Through molecular mechanisms that have not yet been fully elaborated (Gispen, 1986; Alexander et al., 1988), GAP43 is thought to contribute directly to the outgrowth of neuronal processes (Zuber et al., 1989). While first recognized for its increased synthesis and axonal transport in neurons responding to peripheral nerve injury (Skene, 1984; Bisby, 1988), GAP43 and its mRNA are also detectable in some regions of the normal brain (Benowitz et al., 1988; Neve et al., 1988). More GAP43 immunoreactivity has been detected in unmyelinated than myelinated axons both in normal rat sciatic nerves and in the proximal stump of transected nerves (Tetzlaff et al., 1989) and intense immunohistochemical staining has been observed in varicose axons innervating the vasa vasorum of the sciatic nerve and in unmyelinated axons of the myenteric plexus (McGuire et al., 1988; Sharkey et al., 1989). Thus, GAP43 normally appears to be heterogeneously distributed in both the peripheral and central nervous system.

The signals following nerve injury that trigger an increased synthesis of GAP43 (Bisby, 1988) and a regenerative propensity (McQuarrie and Grafstein, 1973; Richardson and Verge, 1986; Molander et al., 1988) in nerve cell bodies are not well understood. With respect to nerve growth factor (NGF) as a possible signal, the observations are paradoxical: both regenerative behavior and its marker, GAP43, are induced in vitro by NGF (Karns et al., 1987; Federoff et al., 1988) yet also induced in vivo by axotomizing lesions that restrict the normal supply of NGF to neurons. However, it has not been proven that all sensory neurons respond in the same way to axotomy: only some sensory neurons have high-affinity NGF receptors (Verge et al., 1989a, b), and it might be speculated that injured NGFresponsive and NGF-unresponsive neurons differ in GAP43 synthesis and conditioning responses.

In experiments to be described, expression of the GAP43 gene was studied in normal and injured rat primary sensory neurons by in situ hybridization with a cDNA probc. Emphasis was placed on heterogeneity among sensory neurons in GAP43 mRNA content and on possible correlations between GAP43 and the high-affinity NGF receptor.

\section{Materials and Methods}

Animal surgery. Experiments were performed on 26 adult female Sprague-Dawley rats weighing approximately $200 \mathrm{gm}$. In some rats, the right sciatic nerve was transected with microsurgical technique under 


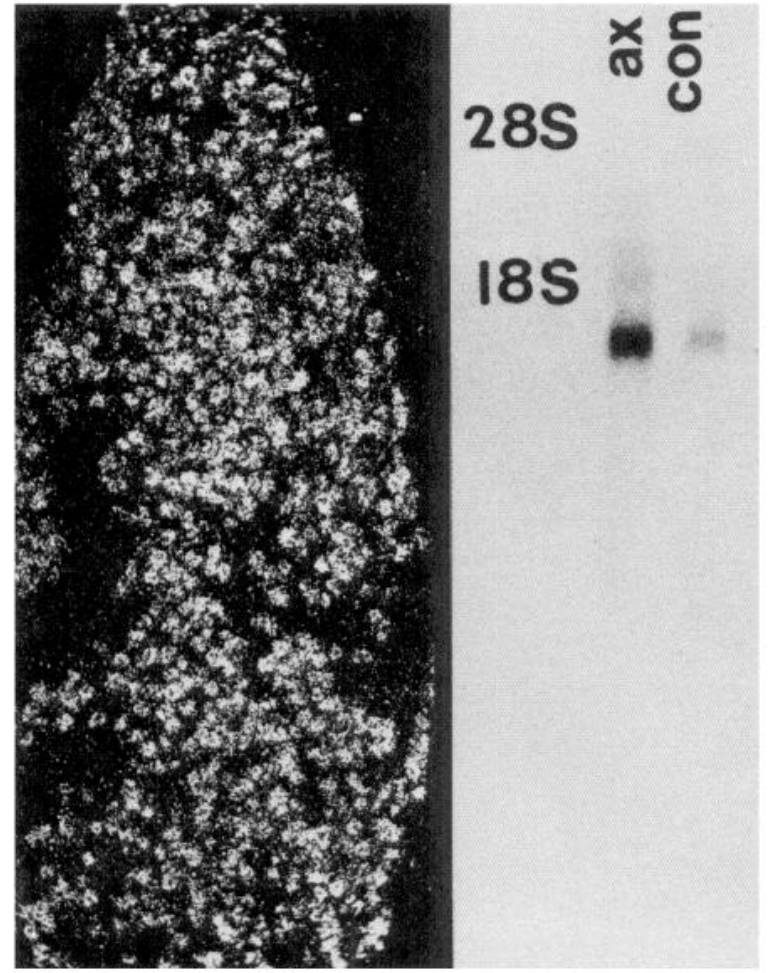

Figure 1. Left, Dark-field photomicrograph of GAP43 hybridization to rat superior cervical ganglion. $\times 55$. Right, Northern blot preparation of total cytoplasmic RNA from control $\mathrm{L}_{\varsigma}$ DRG (con) and DRG associated with axonal transection $5 \mathrm{~d}$ previously $(a x)$. The filter was hybridized with ${ }^{35} \mathrm{~S}-\mathrm{GAP} 43 \mathrm{cDNA}$ which recognizes a single mRNA of $1.4 \mathrm{~kb}$ and much greater manifestation following injury.

pentobarbital anesthesia $(50 \mathrm{mg} / \mathrm{kg}$, i.p.). To expose the sciatic nerve at its origin from the fourth and fifth lumbar $\left(\mathrm{L}_{4}, \mathrm{~L}_{5}\right)$ spinal nerves, a small amount of bone was rongeured from the iliac crest. The sciatic nerve was cut, and a $5 \mathrm{~mm}$ segment was removed. In some instances, $\beta$-NGF from male mouse submandibular glands (Mobley et al., 1976; Chapman et al., 1981) was infused into the lumbar subarachnoid space at $125 \mathrm{ng} /$ hr. For this purpose, Silastic tubing, 0.012 inches in diameter (DowCorning), was connected to an osmotic pump (Alza model 2001) and passed through the dura and arachnoid at the lumbosacral junction so that a $1.5 \mathrm{~cm}$ segment lay intrathecally. The pump was secured to the lumbar fascia. Tissues were analyzed from normal rats and rats that had undergone sciatic nerve transection 1 week previously with or without subsequent NGF infusion. Rats were deeply anesthetized with pentobarbital and perfused per aorta with cold PBS ( $\mathrm{pH}$ 7.4). The $\mathrm{L}_{5}$ DRG and/or superior cervical ganglion were removed, quickly frozen in isopentane, and stored at $-80^{\circ} \mathrm{C}$ for several days. In rats with cut sciatic nerves, the right and left $\mathrm{L}_{5}$ DRG were frozen in the same block of Tissue-Tek (Miles Laboratories) so that they could be processed on the same slide under identical conditions.

Northern blotting. Total cytoplasmic RNA was prepared from 4 pooled DRG $\left(\mathrm{L}_{4}, \mathrm{~L}_{5}\right)$ according to a standard technique (Schibler et al., 1980). Equal amounts of RNA were fractionated by electrophoresis on $1.2 \%$ agarose gels in the presence of $1 \mathrm{M}$ formaldehyde (Maniatis et al., 1982) and blotted onto nitrocellulose by capillary transfer. The filters were covered for $2 \mathrm{hr}$ at $43^{\circ} \mathrm{C}$ in prehybridization solution containing $50 \%$ formamide, $5 \times$ Denhardt's solution, salmon sperm DNA and tRNA $(250 \mu \mathrm{g} / \mathrm{ml})$, DTT (100 mM), 0.2\% SDS, $75 \mathrm{~mm} \mathrm{NaCl}, 25 \mathrm{~mm}$ EDTA in $25 \mathrm{~mm}$ PIPES buffer, $\mathrm{pH} 6.8$. Hybridization for $12-14 \mathrm{hr}$ again at $43^{\circ} \mathrm{C}$ was performed using the same solution with the addition of a ${ }^{35} \mathrm{~S}$ labeled GAP43 cDNA probe $\left(2 \times 10^{6} \mathrm{cpm} / \mathrm{ml}, 10-20 \mathrm{ng} / \mathrm{ml}\right)$, which was generated by random oligonucleotide primed labeling (Feinberg and Vogelstein, 1983) of an isolated cDNA insert (Basi et al., 1987). Posthybridization washes were carried out with $4 \times, 2 \times, 1 \times, 0.5 \times$, and $0.1 \times$ $\mathrm{SSC}$ at $45^{\circ} \mathrm{C}$. Dried filters were exposed to Kodak X-omat $\mathrm{x}$-ray film for 1-2 weeks and developed.
In situ hybridization. Cryostat sections, $5 \mu \mathrm{m}$ thick, were thaw-mounted on gelatin-coated slides, postfixed in phosphate-buffered $4 \%$ paraformaldehyde and treated for prehybridization and hybridization as previously described (Miller et al., 1987). After fixation, the sections were washed in PBS, treated with proteinase $\mathrm{K}(10 \mathrm{ng} / \mathrm{ml})$ for $8 \mathrm{~min}$ at room temperature, fixed again for $5 \mathrm{~min}$ in $4 \%$ paraformaldehyde, and dehydrated in 70,90 , and $100 \%$ ethanol in $0.3 \mathrm{~m}$ sodium acetate. After brief drying, the sections were prehybridized, hybridized, and washed with the same solutions used for Northern blotting. Sections were dipped in Kodak $\mathrm{NTB}_{2}$ emulsion, diluted 1:1 in water, exposed in the dark for 2-3 weeks, and developed. Control slides were hybridized with ${ }^{35} \mathrm{~S}$ labeled plasmid DNA rather than GAP43 cDNA.

$N G F$-receptor radioautography. Unfixed cryostat sections adjacent to those used for GAP43 hybridization were incubated for $90 \mathrm{~min}$ at room temperature with 50 pM ${ }^{125}$ I-NGF, postfixed, defatted, dipped in emulsion, exposed in the dark for $4 \mathrm{~d}$, developed, and stained. As described previously (Verge et al., 1989a), this technique is designed to detect high-affinity NGF binding with half-maximal saturation by picomolar concentrations of NGF.

Histochemistry. Histochemical procedures for detecting somatostatin immunoreactivity and acid phosphatase enzyme activity in postfixed tissue sections have been previously described in detail (Verge et al., 1989 b). For immunohistochemistry, sections were postfixed in buffered formaldehyde/picric acid, pretreated with hydrogen peroxide to deplete endogenous peroxidase, incubated overnight with antisomatostatin-28 (S309 serum generously provided by Dr. Robert Benoit) and processed with avidin-biotin reagents and HRP histochemistry. For acid phosphatase localization, sections were postfixed, rinsed, incubated in a solution containing thiamine monophosphate chloride and lead nitrate, and developed with a sodium sulfide solution.

Image analysis. To correlate GAP43 hybridization and high-affinity NGF binding sites, montages were prepared from adjacent sections and each neuron with a visible nucleolus in 1 of the 2 sections was identified and numbered in both sections (Verge et al., 1989b). Then, NGF-receptor radioautography and GAP43 hybridization were quantified by computer-assisted image analysis (Richardson et al., 1989) of individual neurons viewed under oil-immersion light microscopy. By tracing of the periphery of identified neurons, the cross-sectional area and fraction of area covered by silver grains were measured. To obtain the fraction of cytoplasm covered by silver grains $(f)$, nuclear labeling and area were subtracted from total area and labeling. The fraction $f /(1-f)$ designated "labeling density" was used to correct for grain overlap (Richardson et al., 1989).

Areas were obtained for the same neurons in both preparations and corrected for $8 \%$ linear shrinkage in receptor radioautographs: the greater of the 2 measurements was taken as the true area and the diameter was calculated on the assumption that neurons were spherical. To avoid negative numbers and permit logarithmic conversion, total labeling was used without subtraction of background labeling. Labeling densities were normalized by reference to background labeling obtained by measuring grain densities over several areas of the ganglia devoid of nerve cell bodies. Statistical analyses were performed by computer according to published algorithms (Press et al., 1988).

\section{Results}

Northern blots of DRG (Fig. 1) showed that the probe was recognizing a single mRNA species of $1.4 \mathrm{~kb}$ (Basi et al., 1987) and that the mRNA in DRG increased substantially following sciatic nerve transection. For the superior cervical ganglion, where most neurons bear high-affinity NGF binding sites (Richardson et al., 1986), in situ hybridization yielded strong labeling over virtually all neurons (Fig. 1). Hybridization of radiolabeled plasmid DNA to $\mathrm{L}_{5}$ DRG gave no specific labeling.

\section{Normal DRG}

Labeling with GAP43 cDNA was extremely heterogeneous among neurons in normal $\mathrm{L}_{5}$ DRG (Fig. 2), ranging from less than background to almost 100 times background (Fig. 3) in most preparations. Histograms of labeling densities (Fig. 3) for preparations from 5 normal DRG did not suggest a single neuronal population but rather the existence of 2 normally distrib- 
Figure 2. Photomicrographs of adjacent sections, $5 \mu \mathrm{m}$ thick, from $\mathrm{L}_{\text {s }}$ DRG processed for GAP43 hybridization (left) or NGF-receptor radioautography (right). Note that 7 neurons are heavily labeled and 3 large neurons are lightly labeled with each of the 2 markers. $\times 700$.

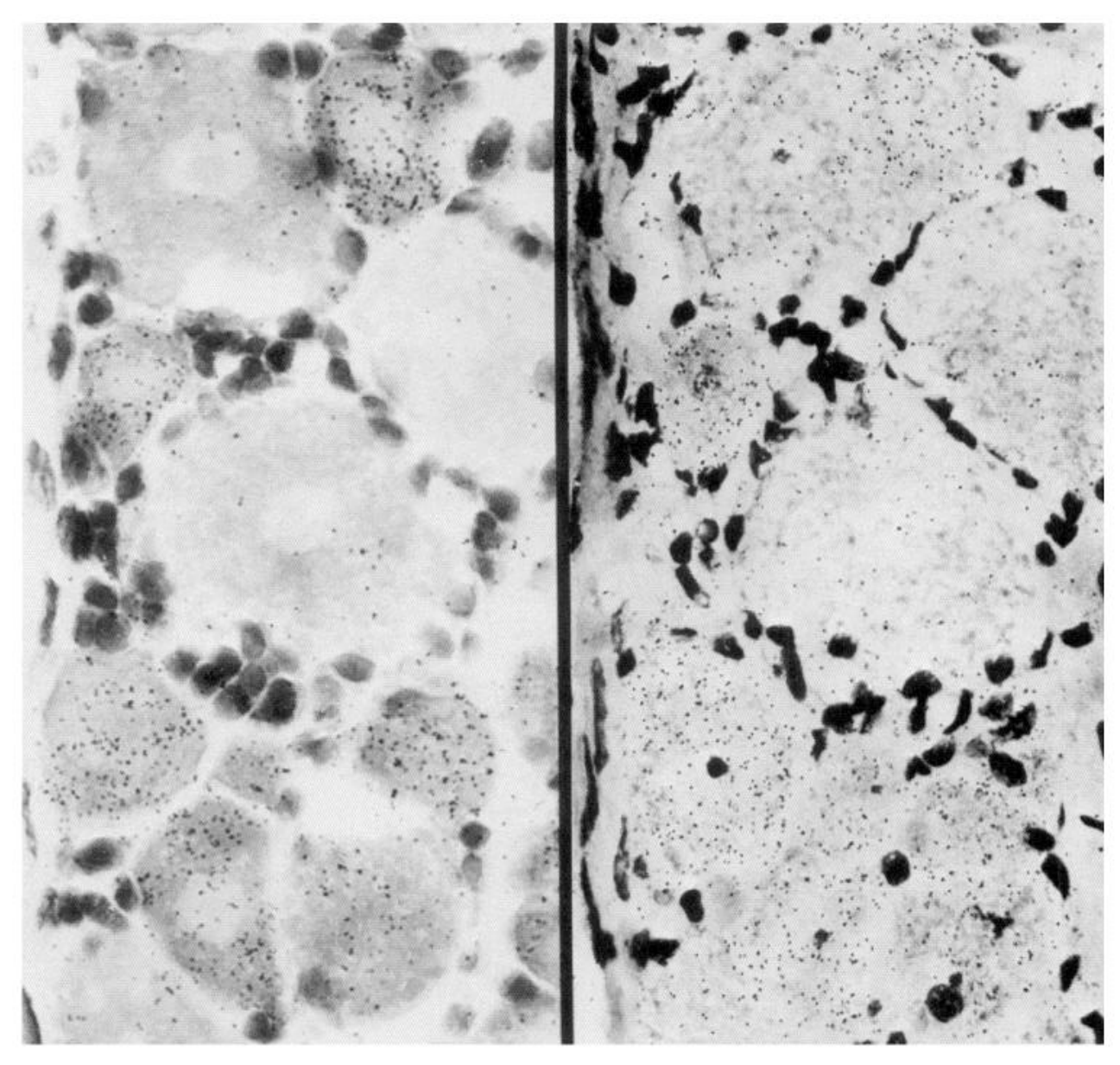

uted populations (on a logarithmic scale) with mean labeling densities in the order of 2-3 times background and 5-20 times background. Labeling tended to be denser in small rather than large neurons (Fig. 4), although many small neurons were lightly labeled and some large neurons were heavily labeled.

As in earlier studies, histograms of the binding of ${ }^{125}$ I-NGF to $L_{5}$ DRG neurons could be fitted to 2 normal curves with 50$60 \%$ and $40-50 \%$ of the neurons (Verge et al., 1989a, b). Evidence has been presented that these 2 curves represent neurons without and with high-affinity NGF receptors (Verge et al., $1989 \mathrm{~b})$. Inspection of groups of sensory neurons suggested a correlation between labeling with NGF and GAP43 mRNA (Fig. 2 ). To verify this impression, detailed quantitative analysis was performed on pairs of adjacent sections from 4 normal DRG. One ganglion was from an unoperated rat, and 3 were left $\mathrm{L}_{5}$ DRG removed 1 week after right sciatic nerve transection. No difference was noted between the true normal ganglion and other 3 control ganglia. In log-log plots, GAP43 labeling densities were obviously correlated to NGF labeling densities (Pearson coefficient $=0.34-0.50, p<0.00001)$. When the thirds of total neuronal population with lightest and heaviest NGF labeling were selected to represent neurons without and with high-affinity receptors (Table 1), mean densities of GAP43 labeling for the receptor-bearing populations were 2.3-4.4 times greater than those for the receptor-lacking populations $(p<0.0001$, Student's $t$ test). Most neurons could be placed into 1 of 2 clusters (Fig. 3)-lightly or heavily labeled by both procedures. Virtually all neurons with dense ${ }^{125}$ I-NGF labeling also had high concen- trations of GAP43 mRNA, and most neurons with light NGF labeling had little GAP43 mRNA. However, 3-7\% of all neurons fell in the subpopulation defined arbitrarily by NGF labeling in the lower third and GAP labeling in the upper third.

In another $\mathrm{L}_{5}$ DRG, GAP43 hybridization was examined for neurons with somatostatin immunoreactivity and acid phosphatase enzyme activity: both groups have previously been shown to lack high-afffinity NGF receptors (Verge et al., 1989b). Somatostatin-positive and phosphatase-positive neurons were invariably small and lightly labeled in GAP43 hybridization (Fig. 4).

\section{Nerve transection and NGF infusion}

In rats killed 1 week after right sciatic nerve transection, labeling by GAP43 hybridization was consistently greater in the right than left $L_{5}$ DRG. This observation was made for 4 rats by inspection under dark-field illumination of pairs of sections, mounted on single slides (Fig. 5). In 2 rats, NGF labeling densities and GAP43 labeling densities were quantified for both right and left $\mathrm{L}_{5}$ DRG. In both cases, the mean NGF labeling density was significantly reduced (by 25 and $18 \%$ ) and the mean GAP43 labeling was significantly increased (to 2.0 and 2.7 times normal). In histograms of GAP43 labeling densities in DRG associated with a cut sciatic nerve (Fig. 5), most values fell within a single normal curve except for $5-10 \%$ of the total which remained at lower values. When GAP43 labeling densities were plotted against NGF labeling densities in scatter diagrams for individual neurons or as 3-dimensional histograms, no corre- 

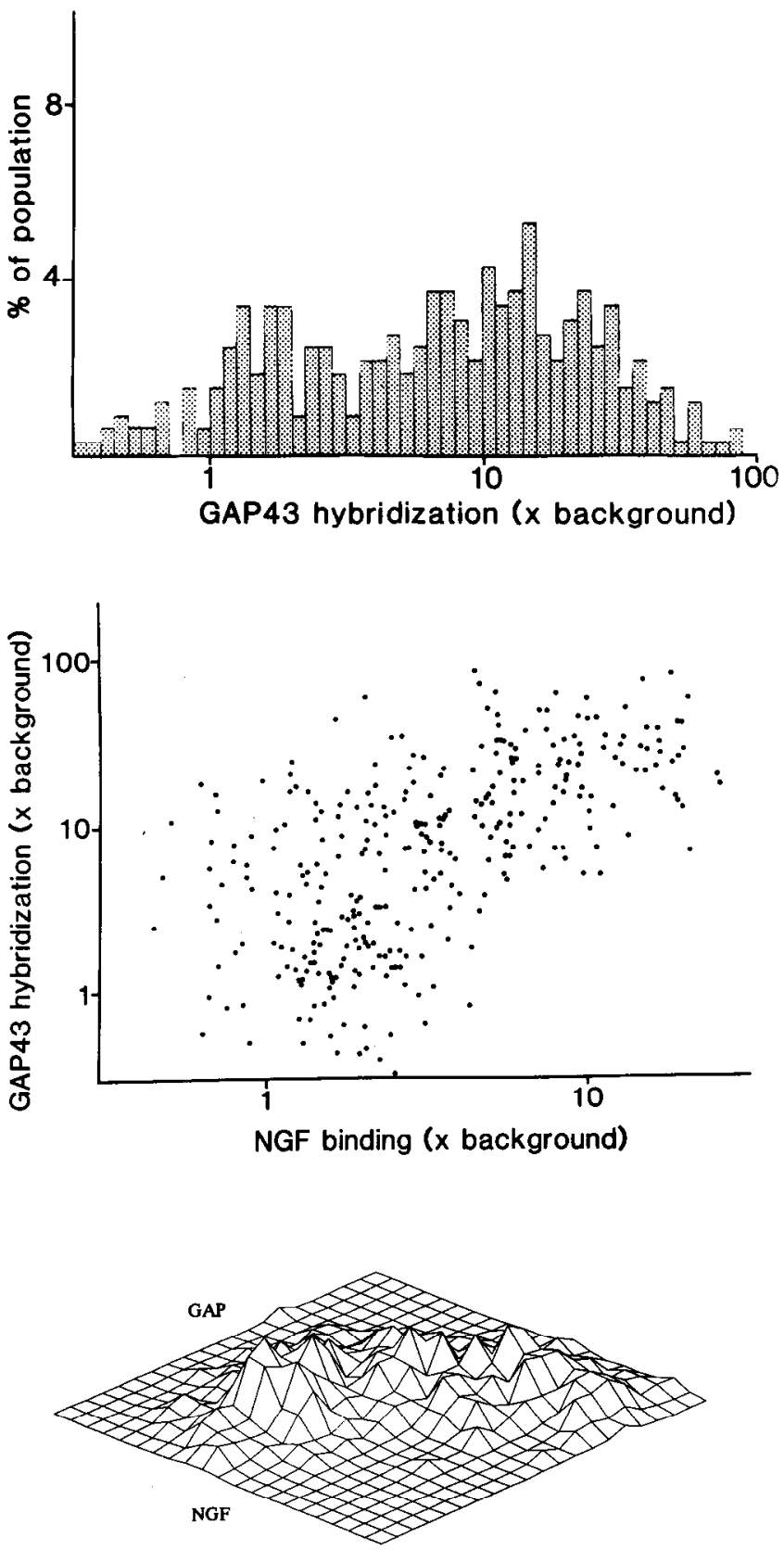

Figure 3. The 3 diagrams all present data from 322 neurons identified and quantified for binding of ${ }^{125}$ I-NGF and hybridization with ${ }^{35} \mathrm{~S}$ GAP43 cDNA. The histogram of GAP43 labeling densities (upper) shows a bimodal distribution compatible with the existence of 2 populations. The scatter diagram (middle) and 3-dimensional histogram (lower) are derived from labeling densities for individual neurons in the 2 preparations. Note that most neurons fall within 2 clusters, "labeled" or "unlabeled" by the 2 markers.

lation between the 2 labeling densities was detected. Mean GAP43 labeling densities for the thirds of the population most heavily and lightly labeled by NGF were almost identical. In short, 1 week after sciatic nerve transection, the concentrations of GAP43 mRNA were homogeneously high in almost all sensory neurons.

Three animals were examined 1 week after right sciatic nerve transection followed by continuous infusion of NGF into the
Table 1. Mean GAP43 labeling density $(x$ background)

\begin{tabular}{lrcr} 
Animal number & \multicolumn{1}{c}{$\begin{array}{l}\text { Total } \\
\text { population }\end{array}$} & $\begin{array}{l}\text { Lightly labeled } \\
\text { by NGF }\end{array}$ & \multicolumn{1}{l}{$\begin{array}{l}\text { Heavily labeled } \\
\text { by NGF }\end{array}$} \\
\hline 1 (normal) & $13.2 \pm 0.9$ & $5.3 \pm 0.6$ & $23.1 \pm 1.5$ \\
2 (normal) & $9.8 \pm 0.6$ & $4.8 \pm 0.6$ & $18.5 \pm 1.1$ \\
3 (normal) & $19.0 \pm 1.3$ & $8.3 \pm 1.3$ & $36.7 \pm 2.5$ \\
4 (normal) & $5.0 \pm 0.3$ & $3.4 \pm 0.3$ & $8.0 \pm 0.6$ \\
3 (cut) & $38.4 \pm 2.0$ & $43.6 \pm 3.8$ & $40.9 \pm 3.5$ \\
4 (cut) & $13.5 \pm 0.5$ & $13.4 \pm 0.8$ & $13.6 \pm 0.9$
\end{tabular}

GAP43 labeling densities were calculated for the thirds of normal neurons mos lightly and heavily labeled by NGF in normal $L_{s}$ DRG from 4 different rats. Also included is the comparable information for 2 ganglia associated with the sciatic nerve transection, 1 week previously. Data from animal 1 and animal 4 are displayed in Figures 2 and 5, respectively (means \pm SEM; $n=243-327$ ).

subarachnoid space. Intrathecal infusion of NGF counteracted the loss of NGF-binding sites that usually follows nerve transection more reliably than application of NGF to the cut nerve stump (Verge et al., 1989a). In the contralateral $L_{5}$ DRG associated with an intact sciatic nerve, most neurons fell into 1 of 2 clusters-either heavily or lightly labeled by both procedures (Fig. 6). NGF infusion seemed to accentuate both GAP43 and NGF labeling in the heavily labeled subpopulation, so that, in 3-dimensional histograms, the 2 clusters of neurons were more widely separated than in normal ganglia. Following sciatic nerve transection and continuous infusion of NGF, the distribution of GAP43 labeling densities in the ipsilateral $L_{5} D R G$ conformed to a single peak with uniformly high labeling: the pattern was similar to that after simple transection.

\section{Discussion}

\section{Technical considerations}

The presence of a single band on Northern blots and the absence of any hybridization with labeled plasmid vector indicate that the in situ hybridization is indeed recognizing GAP43 mRNA. It is not known whether changes in GAP43 mRNA reflect changes in transcription or in mRNA stability.

The procedures for quantification currently used allow comparison of labeling by GAP43 cDNA among groups of neurons in the same section or in different sections on the same slide. Because of slide-to-slide variability due to temperature dependence of the GAP43 hybridization, probe concentration, and other unknown factors, we have not compared labeling densities from sections processed on different slides. Neither have we attempted to convert labeling densities to absolute values of cytoplasmic mRNA per cell. This difficult task would require rigorous control of conditions plus standardizing hybridizations with known amounts of GAP43 mRNA.

\section{Correlation between GAP43 and NGF binding in normal ganglia}

Among normal rat DRG neurons, expression of the GAP43 gene is strongly correlated with deployment of the high-affinity NGF receptor. Given that NGF can influence GAP43 synthesis in pheochromocytoma cells (Karns et al., 1987; Federoff et al., 1988), it seems plausible to attribute the high basal synthesis of GAP43 in sympathetic neurons and some sensory neurons to continuous stimulation by endogenous NGF. An alternative possibility is that periodic mechanical injury to the exposed cutaneous terminals of some primary sensory neurons stimulates a mild manifestation of the cell body response to axotomy: 


\section{T M Pase}

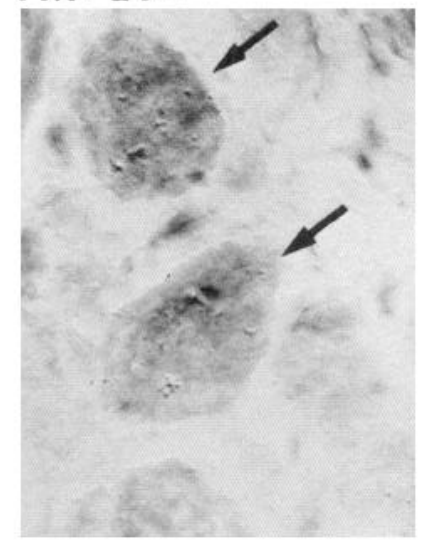

\section{Som}

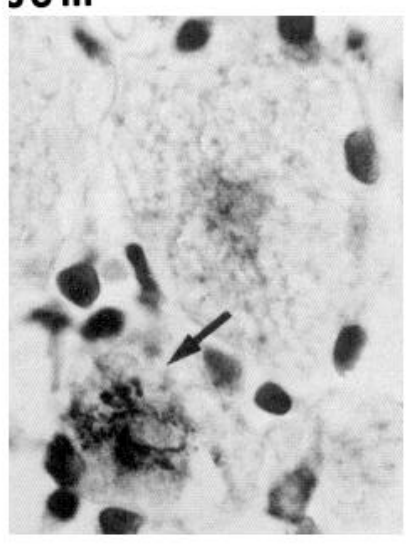

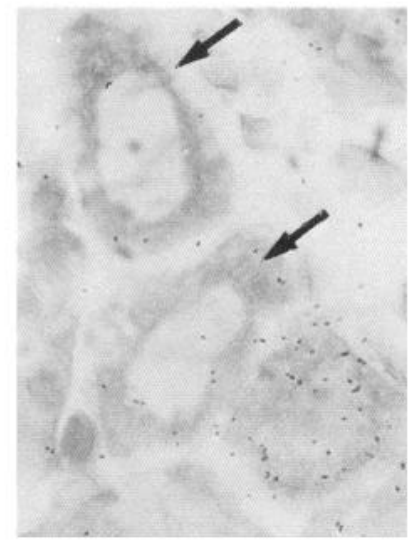

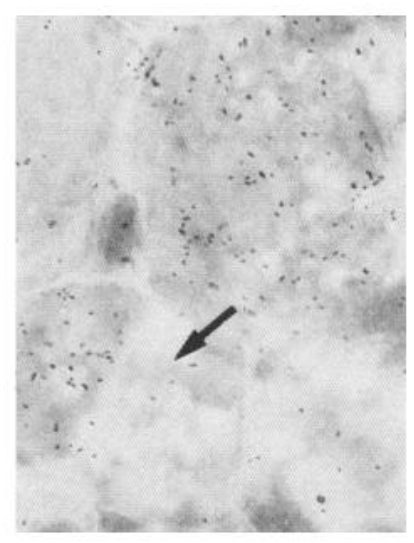

Total population

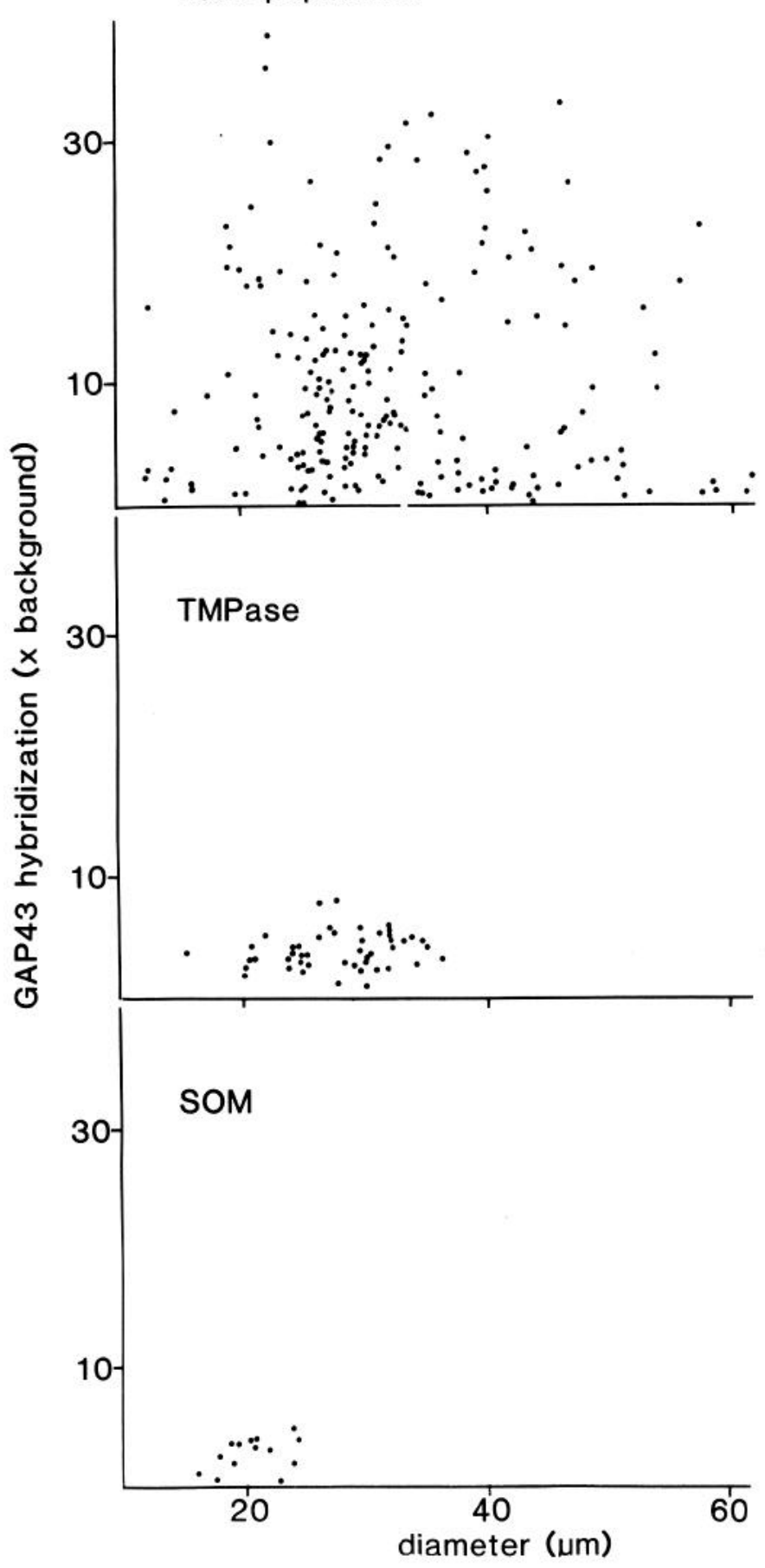

Figure 4. Upper left, Photomicrographs of adjacent sections of $\mathrm{L}_{5}$ DRG processed to show thiamine monophosphatase activity (left) or GAP43 mRNA (right). Note that the 2 neurons with thiamine monophosphatase activity have little GAP43 mRNA. $\times 810$. Lower left, Photomicrographs of adjacent sections processed for somatostatin immunohistochemistry (left) or GAP43 hybridization (right). Note that the somatostatin-positive neuron is GAP43-negative. $\times 810$. Upper right, Scatter diagram in which GAP43 labeling density is plotted against diameter for 223 neurons representing the entire $\mathrm{L}_{5}$ DRG. There are wide ranges of size and labeling density with little correlation between the 2 measurements. Middle right, Individual neurons were identified to have acid phosphatase acitivy and quantified for GAP43 hybridization on the adjacent section. Note that all the neurons with thiamine phosphatase reaction product are small with little GAP43 labeling $(n=48)$. Lower right, Quantification of GAP43 labeling density and size for neurons seen in the adjacent section to have somatostatin immunoreactivity. Somatostatin positive neurons are small with GAP43 labeling near background $(n=14)$.

degenerated terminals have been observed in the subepidermal C fiber network of normal rats (Jackson and Diamond, 1984). However, this hypothesis seems highly unsatisfactory for sympathetic neurons in the superior cervical ganglia, where GAP43 is abundantly synthesized even though their axon terminals are minimally exposed to chronic injury. We favor the suggestion that NGF is causally involved in the high basal rates of GAP43 synthesis by some neurons. 

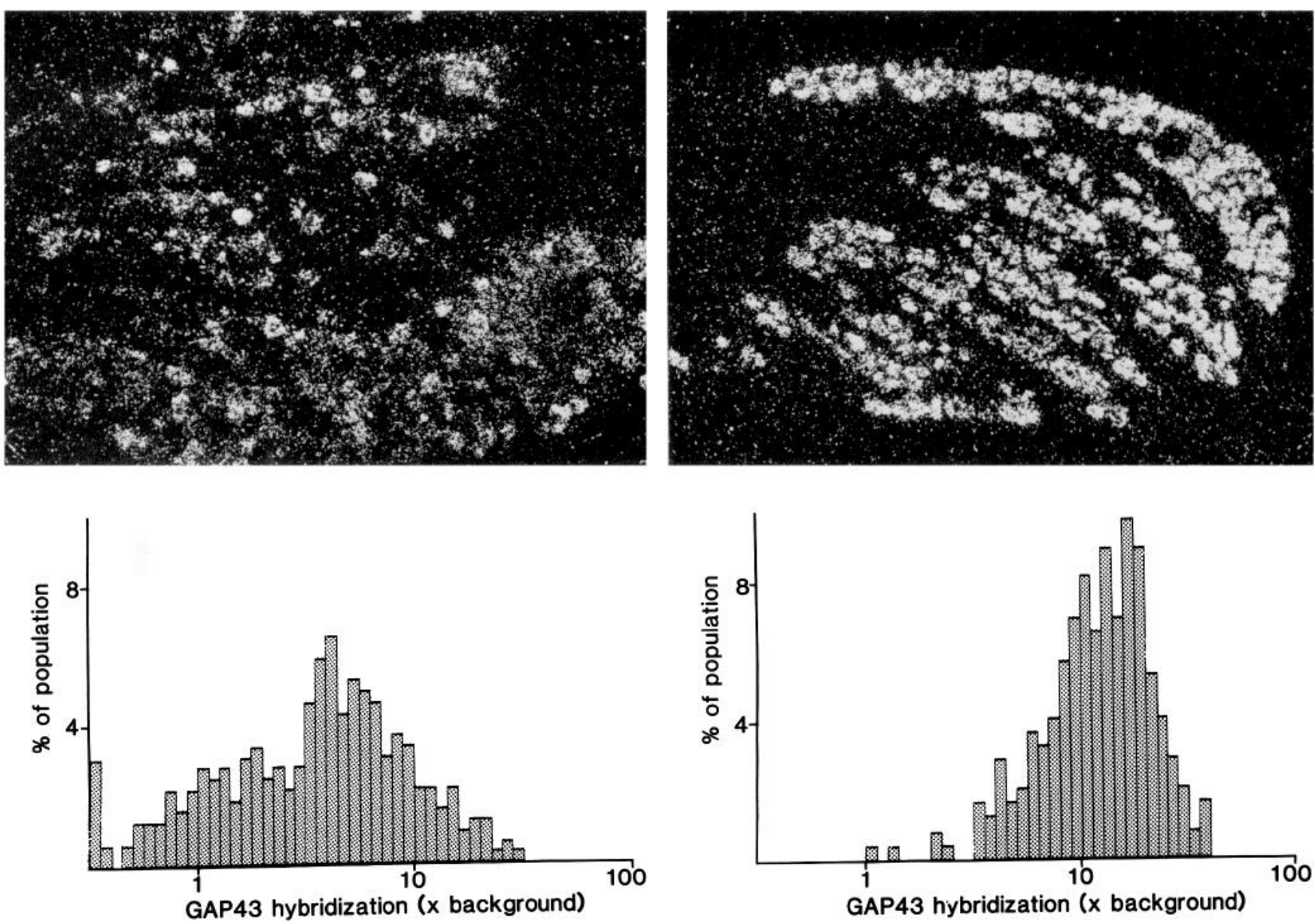

INTACT

CUT
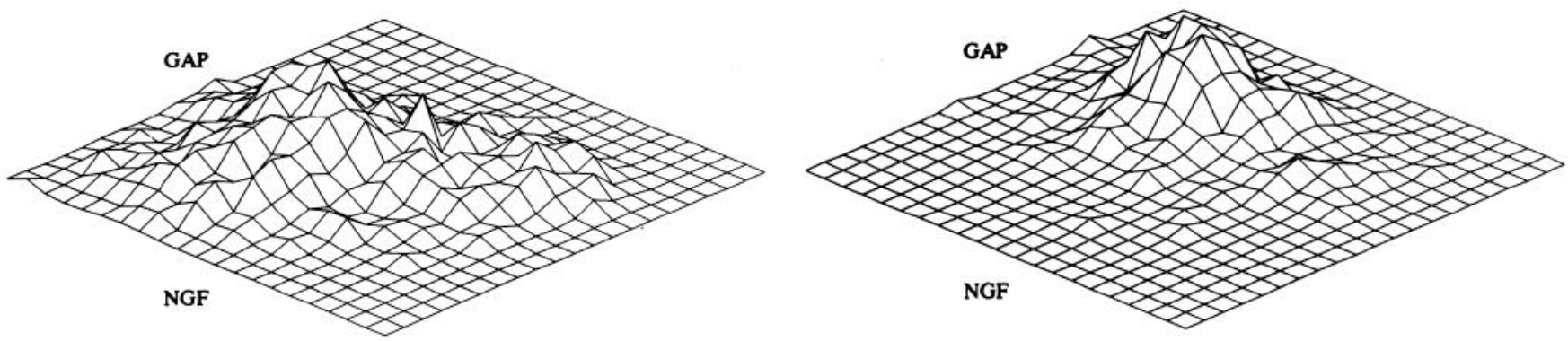

Figure 5. One week after sciatic nerve transection the left and right $\mathrm{L}_{5} \mathrm{DRG}$ were removed. Data on the left and right are for the left and right DRG, respectively. Upper, Dark-field photomicrographs of sections after GAP43 in situ hybridization. Note the heterogeneous labeling in the normal ganglia and the increased homogeneous labeling associated with nerve transection. $\times 55$. Middle, Histograms of GAP43 labeling densities showing a bimodal distribution in the left DRG and a single densely labeled peak in the right DRG. Lower, Three-dimensional histograms of NGF and GAP43 labeling. In the normal ganglion $(n=327)$, note the correlation between the 2 labels: After nerve transection $(n=248)$, this correlation is lost and GAP43 labeling densities appear to be uniformly high.

Virtually all substance P-immunoreactive neurons and most CGRP-immunoreactive neurons have high-affinity NGF binding sites (Verge et al., 1989b), and neurons with significant highaffinity binding invariably are labeled by GAP43 cDNA (Fig 3). Therefore, it can be assumed that neurons with substance $P$ immunoreactivity and strong CGRP immunoreactivity synthesize GAP43 at high basal level.
Small sensory neurons with somatostatin immunoreactivity or acid phosphatase enzyme activity have been shown previously to lack high-affinity NGF binding sites (Verge et al., 1989b), and these neurons are shown here to have low basal levels of GAP43 mRNA. In most normal DRG, a few small neurons were found with little NGF binding but relatively high GAP43 hybridization: these neurons could represent a true subpopu- 

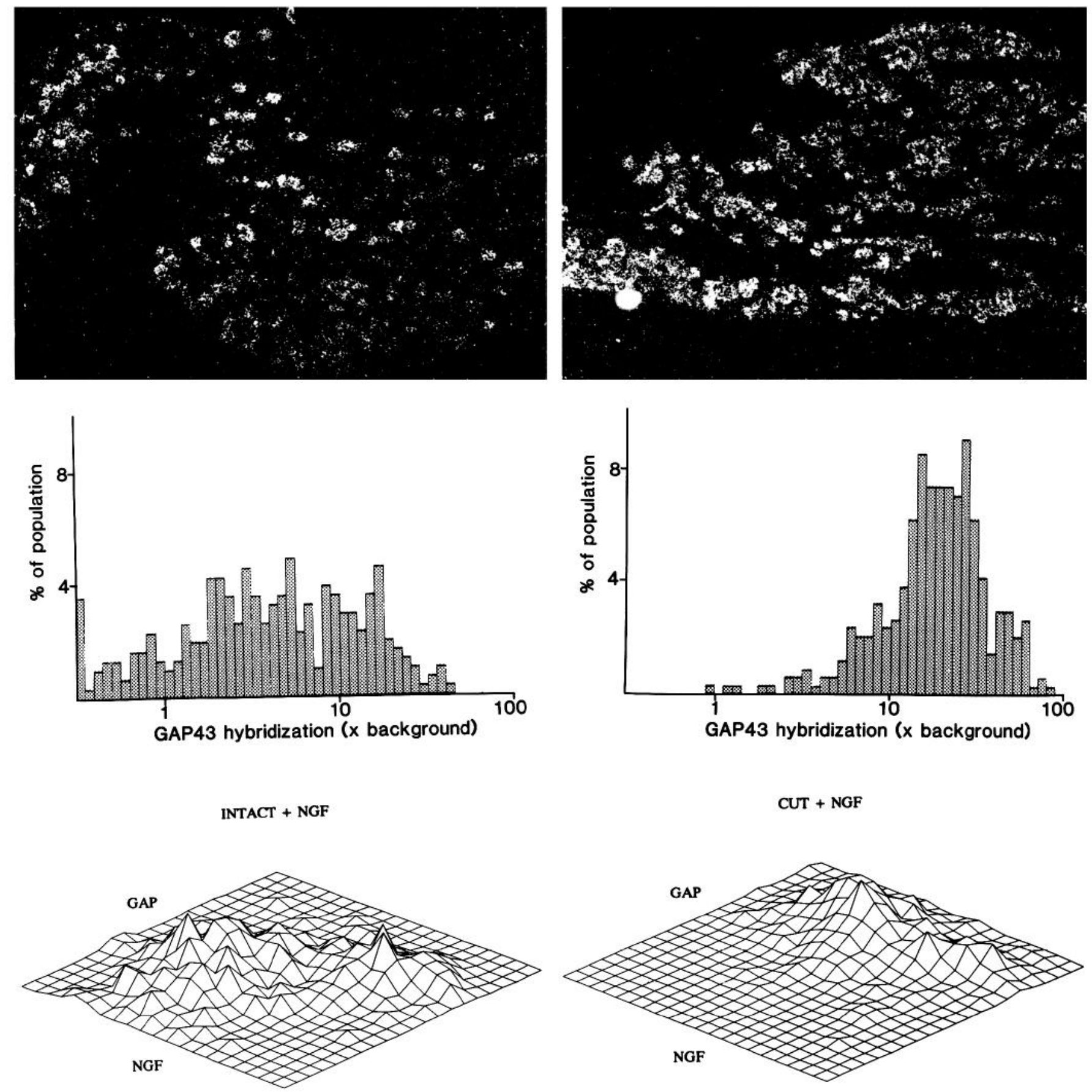

Figure 6. Data for left and right $\mathrm{L}_{5}$ DRG removed 1 week after right sciatic nerve transection followed by continuous infusion of NGF. Upper, Dark-field photomicrographs of sections after GAP43 in situ hybridization. Labeling appears to be supranormal but is still heterogeneous in the left DRG and homogeneously high in the right DRG. $\times 55$. Middle, Histograms of GAP43 labeling densities showing a bimodal distribution (left) and unimodal distribution (right). The patterns are similar to those in Figure 5. Lower, Three-dimensional histograms of GAP43 and NGF labeling showing once again a correlation between the 2 indices when the sciatic nerve is intact $(n=309)$ and a uniform increase of GAP43 labeling after the nerve has been cut $(n=347)$.

lation or falsely high GAP43 labeling of small cross-sectional areas.

One speculation is that high basal concentration of GAP43 confers upon some DRG neurons a capacity for collateral sprouting. After injury to a peripheral nerve, some functionally defined subclasses of sensory axons from an adjacent nerve can grow into the denervated territory (Jackson and Diamond, 1984). The fact that such sprouting is blocked by antiserum to NGF
(Diamond et al., 1987) suggests the involvement of NGF and participation of NGF-receptor-bearing neurons: the latter population is shown here also to express the GAP43 gene. By similar conjecture, the outgrowth of uninjured sympathetic neurons in response to partial denervation (Kennedy and Sakuta, 1984; Kessler, 1985) might also depend upon the presence of GAP43 within neurons and some local stimulus from denervated Schwann cells or target cells. On the other hand, uninjured 
motoneurons are also capable of sprouting (Brown et al., 1981) yet have uniformly low basal levels of GAP43 (Tetzlaff and Bisby, 1988). The possible contribution of GAP43 to sprouting might be clarified by better histochemical definition of sensory axons that are capable of collateral sprouting. The possibility that GAP43 has some unrecognized function in a subpopulation of sensory neurons must also be entertained.

\section{Changes after injury}

One week following peripheral nerve transection, the concentration of GAP43 mRNA is increased in most $L_{5}$ DRG neurons. The few neurons with low GAP43 labeling after injury are assumed to project into the dorsal cutaneous nerve (Diamond et al., 1987) and not the sciatic nerve. Although sensory neurons differ in some of their degenerative responses to axonal injury (Aldskogius and Risling, 1983), the present observations indicate that synthesis of GAP43 is induced to a high level in all or almost all sensory neurons shortly after their axons are cut. It remains to be proved that sensory nerve cell bodies are conditioned to the same extent or maintain high levels of GAP43 for the same period of time.

One week after nerve transection, GAP43 synthesis is increased in neurons with or without high-affinity NGF receptors, and under conditions of reduced retrograde supply of NGF or continuous infusion of NGF. These observations indicate that some signal other than NGF or lack of NGF is responsible for the early posttraumatic induction of GAP43. Both increased axonal transport of GAP43 and a propensity for regeneration result directly or indirectly from the loss of some retrograde signal normally arising distal to the site of axonal injury (Benowitz et al., 1983; Richardson and Verge, 1986; Bisby, 1988). Because NGF counteracts some of the changes of axotomized sensory neurons (Fitzgerald et al., 1985; Rich et al., 1987; Verge et al., 1989a) but not the increasc in GAP43, it seems necessary to invoke at least 2 molecular signals to explain the multiple nerve cell responses to injury.

\section{References}

Aldskogius, H., and M. Risling (1983) Preferential loss of unmyelinated $\mathrm{L}_{7}$ dorsal root axons following sciatic nerve resection in kittens. Brain Res. 289: 358-361.

Alexander, K. A., B. T. Wakim, G. S. Doyle, K. A. Walsh, and D. R. Storm (1988) Identification and characterization of the calmodulinbinding domain of neuromodulin, a neuro-specific calmodulin-binding protein. J. Biol. Chem. 263: 7544-7549.

Basi, G. S., R. D. Jacobson, T. Virag, J. Schilling, and J. H. P. Skene (1987) Primary structure and transcriptional regulation of GAP43, a protein associated with nerve growth. Cell 49: 785-791.

Benowitz, L. I., and A. Routtenberg (1987) A membrane phosphoprotein associated with neural development, axonal regeneration, phospholipid metabolism and synaptic plasticity. Trends Neurosci. 10: 527-532.

Benowitz, L. I., M. G. Yoon, and E. R. Lewis (1983) Transported proteins in the regenerating optic nerve: Regulation by interactions with the optic tectum. Science 222: 185-188.

Benowitz, L. I., P. J. Apostolides, N. Perrone-Bizzozero, S. P. Finklestein, and $H$. Zwiers (1988) Anatomical distribution of the growthassociated protein GAP43/B50 in the adult rat brain. J. Neurosci. 8: 339-352.

Bisby, M. A. (1988) Dependence of GAP43 (B50,F1) transport on axonal regeneration in rat dorsal root ganglion neurons. Brain Res. 458: $157-161$.

Brown, M. C., R. L. Holland, and W. G. Hopkins (1981) Motor nerve sprouting. Annu. Rev. Neurosci. 4: 17-42.

Chapman, C. E., B. E. C. Banks, C. A. Vernon, and J. M. Walker (1981) The isolation and characterization of nerve growth factor from the prostate gland of the guinea-pig. Eur. J. Biochem. 115: 347-351.
Diamond, J., M. Coughlin, L. Macintyre, M. Holmes, and B. Visheau (1987) Evidence that endogenous beta nerve growth factor is responsible for the collateral sprouting, but not the regeneration of nociceptive axons in adult rats. Proc. Natl Acad. Sci. USA 84: 65966600.

Federoff, H. J., E. Grabczyck, and M. C. Fishman (1988) Dual regulation of GAP43 gene expression by nerve growth factor and glucocorticoids. J. Biol. Chem. 263: 19290-19295.

Feinberg, A. P., and B. Vogelstein (1983) A technique for radiolabeling DNA restriction endonuclease fragments to high specific activity. Anal. Biochem. 13:6-13.

Fitzgerald, M., P. D. Wall, M. Goedert, and P. C. Emson (1985) Nerve growth factor counteracts the neurophysiological and neurochemical effects of chronic sciatic nerve section. Brain Res. 332: 131-141.

Gispen, W. H. (1986) Phosphoprotein B-50 and phospho-inositides in brain synaptic plasma membranes: A possible feedback mecha nism. Trans. Biochem. Soc. UK 14: 163-165.

Jackson, P. C., and J. Diamond (1984) Temporal and spatial constraints on the collateral sprouting of low-threshold mechanosensory nerves in the skin of rats. J. Comp. Neurol. 226: 336-345.

Karns, L. R., S. G. Ng, J. A. Freeman, and M. C. Fishman (1987) Cloning of complementary DNA for GAP43, a neuronal growthrelated protein. Science 236: 597-600.

Kennedy, W. R., and M. Sakuta (1984) Collateral reinnervation of sweat glands. Ann. Neurol. 15: 73-78.

Kessler, J. A. (1985) Parasympathetic, sympathetic and sensory interactions in the iris: Nerve growth factor regulates cholinergic ciliary ganglion innervation in vivo. J. Neurosci. 5: 2719-2725.

Maniatis, T., E. F. Fritsch, and J. Sambrook (1982) Molecular Cloning: A Laboratory Manual, Cold Spring Harbor Laboratory, NY.

McGuire, C. B., G. J. Snipes, and J. J. Norden (1988) Light-microscopic immunolocalization of the growth- and plasticity-associated protein GAP43 in the developing rat brain. Dev. Brain Res. 41: $277-$ 291

McQuarrie, I. M., and B. Grafstein (1973) Axon outgrowth enhanced by a previous nerve injury. Arch. Neurol. 29: 53-55.

Miller, F. D., C. C. G. Naus, M. Durand, F. E. Bloom, and R. J. Milner (1987) Isotypes of $\alpha$-tubulin are differentially regulated during neuronal maturation. J. Cell Biol. 105: 3065-3073.

Mobley, W. C., A. Shenker, and E. M. Shooter (1976) Characterization and isolation of proteolytically modified nerve growth factor. Biochemistry 15: 5543-5551.

Molander, C., E. Kiuman, and H. Aldskogius (1988) Expansion of spinal cord primary sensory afferent projection following combined sciatic nerve transection and saphenous nerve crush. J. Comp. Neurol. 276: $436-441$.

Neve, R. L., E. A. Fuich, E. D. Bird, and L. I. Benowitz (1988) Growthassociated protein GAP43 is expressed selectively in associative regions of the adult human brain. Proc. Natl. Acad. Sci. USA 85: 36383642.

Press, W. H., B. P. Flannery, S. A. Teukolsky, and W. T. Vetterling (1988) Numerical Recipes in C, Cambridge University Press, England.

Rich, K. M., J. R. Luszczinski, P. A. Osborne, and E. M. Johnson (1987) Nerve growth factor protects adult sensory neurons from cell death and atrophy caused by nerve injury. J. Neurocytol. 16: 261268.

Richardson, P. M., and V. M. K. Verge (1986) The induction of a regenerative propensity in sensory neurons following peripheral axonal injury. J. Neurocytol. 15: 585-594.

Richardson, P. M., V. M. K. Vcrgc, and R. J. Riopclle (1989) Quantitative radioautography for NGF receptors. In Nerve Growth Factors, R. A. Rush, ed., pp. 315-326, Wiley, New York.

Schibler, U., M. Tosi, A. C. Pittet, L. Fabiani, and P. K. Wellauer (1980) Tissue-specific expression of mouse alpha-amylase genes. J. Mol. Biol. 142: 93-116.

Sharkey, K. A., W. Tetzlaff, P. J. Coggins, H. Zwiers, M. Bisby, and J. S. Davison (1989) Growth associated protein B50 (GAP43) is present in the mammalian enteric nervous system. Soc. Neurosci. Abstr. 15: 301 .

Skene, J. H. P. (1984) Growth-associated proteins and the curious dichotomies of nerve regeneration. Cell 37:697-700.

Tetzlaff, W., and M. A. Bisby (1988) Changes in gene expression following axotomy are similar in rubrospinal (CNS) and facial (PNS) neurons. Soc. Neurosci. Abstr. 14: 805.

Tetzlaff, W., H. Zwiers, K. Lederis, L. Cassar, and M. A. Bisby (1989) 
Axonal transport and localization of B50 GAP43-like immunoreactivity in regenerating sciatic and facial nerves of the rat. J. Neurosci. 9: 1303-1313.

Verge, V. M. K., R. J. Riopelle, and P. M. Richardson (1989a) Nerve growth factor receptors on normal and injured sensory neurons. $J$. Neurosci. 9: 914-922.
Verge, V. M. K., P. M. Richardson, R. Benoit, and R. J. Riopelle (1989b) Histochemical characterization of sensory neurons with highaffinity receptors for nerve growth factor. J. Neurocytol. 18: 583-591.

Zuber, M. X., D. W. Goodman, L. R. Karns, and M. C. Fishman (1989) The neuronal growth-associated protein GAP43 induces filopodia in non-neuronal cells. Science 224: 1193-1195. 